the present technique is also characterized by excellent sensitivity. Signal-to-background ratios of 3:1 or better are seen in CaF, and could probably be greatly improved.

This work was performed under the auspices of the Division of Basic Energy Sciences of the U. S. Department of Energy.

${ }^{1}$ R。W. Field, A. D. English, T。 Tanaka, D. O. Harris, and D. A. Jennings, J. Chem. Phys. 59, 2191 (1973).

${ }^{2}$ P. J. Domaille, T.C. Steimle, and D. O. Harris, J. Mol. Spectrosc。 65 , 354 (1977).

${ }^{3}$ P. J. Domaille, T. C. Steimle, and D. O. Harris, J. Mol. Spectrosc。68, 146 (1977).

${ }^{4}$ R. W. Field, D. O. Harris, and T. Tanaka, J. Mol. Spectrosc. 57, 107 (1975)。

${ }^{5}$ J. Nakagawa, P. J. Domaille, T. C. Steimle, and D. O. Harris, J. Mol. Spectrosc. 70, 374 (1978)。

${ }^{6}$ A. Adams, W. Klemperer, and J. M. Dunn, Can. J. Phys. 46, 2213 (1968).
${ }^{7}$ D. W. Green, Can。J. Phys。49, 2552 (1971)。

${ }^{8}$ I. Renhorn, private communication.

${ }^{9}$ S. D. Rosner, T. D. Gaily, and R. A. Holt, Phys。 Rev. Lett. 35, 785 (1975)。

${ }^{10}$ W. Ertman and P.Hofer, Z. Phys。A 276, 9 (1976).

${ }^{11}$ W. J. Childs, O. Poulsen, and L。 S。 Goodman, Phys. Rev. A 19 , 160 (1979).

${ }^{12}$ W. J. Childs, L. S. Goodman, and O. Poulsen, in Proceedings of the Fourth International Conference on Laser Spectroscopy IV, Rottach-Egern, Germany, 11-15 June 1979, edited by $\mathrm{H}$. Walther and $\mathrm{K} . \mathrm{W}$. Rothe (Springer-Verlag, Heidelberg, 1979), p. 566.

${ }^{13}$ The present instrument is a simplified version of the one described in J. L。 Hall and S. A. Lee, Appl。 Phys. Lett. 29, 367 (1976).

${ }^{14}$ L。 B. Knight, Jr., W。C. Easley, W.Weltner, Jr。, and M. Wilson, J. Chem. Phys. 54, 322 (1971).

${ }^{15}$ W. J. Childs and L。 S. Goodman, to be published.

${ }^{16} \mathrm{G}$. L. Goodman, to be published.

${ }^{17} \mathrm{G}$. Herzberg, Spectra of Diatomic Molecules (Van Nostrand, New York, 1950).

${ }^{18}$ R. S. Frosch and H. M. Foley, Phys. Rev。 $\underline{88}, 1337$ (1952).

${ }^{19}$ N. F. Ramsey, Phys.Rev。 $\underline{76}, 996$ (1949).

\title{
Bounds on Total Cross Sections in Atom-Atom and Atom-Ion Collisions by Geometric Methods
}

\author{
V. $\operatorname{Enss}^{(a)}$ \\ The Institute for Advanced Study, Princeton, New Jersey 08540 \\ and \\ B. Simon \\ Departments of Mathematics and Physics, Princeton University, Princeton, New Jersey 08540 \\ (Received 19 November 1979)
}

\begin{abstract}
A method is presented for obtaining explicit bounds for the total cross section (including scattering into several final charged fragments) for the scattering of two bound clusters of nuclei and electrons so long as either both clusters are neutral or one is neutral and without an electric dipole moment.
\end{abstract}

In this Letter, we want to describe a simple and direct physical approach to the question of obtaining bounds on total cross sections in nonrelativistic quantum mechanics; fuller results and technical details will appear elsewhere. ${ }^{1}$ Among our main results are the following:

(1) If two neutral bound clusters of charged particles ${ }^{2}$ are scattered, total cross sections are finite.

(2) If a neutral bound cluster of charged particles with zero electric dipole moment ${ }^{3}$ is scat- tered from an arbitrary cluster of charged particles, total cross sections are finite.

(3) In two-body potential scattering, we obtain fairly strong bounds on cross sections for arbitrary potentials with support in a ball of radius $R$ depending only on $R$.

(4) In two-body potential scattering, we give upper bounds on the large-coupling-constant behavior of total cross sections which have the correct $^{4}$ asymptotic behavior.

(5) We come to the rather surprising conclu- 
sion that, as far as bounds on total cross sections are concerned, polarization effects are not relevant. We expand on this point below.

We note that our bounds involve total cross sections, i.e., including final states with Coulomb interactions. Our bounds are only on cross sections averaged over a small range of initial velocities but with no averaging over initial directions. Moreover, in cases (1) and (2), the bounds have the correct energy behavior; i.e., averaged over the velocity interval from $v_{0}$ to $v_{0}+\delta v$ we have a $C(\delta v) v_{0}^{-2}$ bound.

Moreover, while we have not computed explicit constants, ${ }^{5}$ our bounds involve only explicitly computable numbers and integrals of potentials. The usual time-independent approach, when applicable, ${ }^{6}$ will not yield explicit bounds, since the Lippmann-Schwinger equation is solved by appealing to a Fredholm alternative and no information ${ }^{7}$ is obtained about the size of solutions.

Our approach is in the first place time dependent, and in this regard it is motivated and heavily influenced by the pioneering work of Amrein and Pearson, ${ }^{8}$ who obtained among other results information about (3) and (4) above, ${ }^{9}$ but upon which we improve. With Sinha, ${ }^{10}$ they obtained results on multiparticle two-cluster scattering in situations allowing long-range forces among the particles within a cluster but only short-range forces between particles in different clusters.
Thus charged particles with Coulomb interaction are allowed in one cluster only. ${ }^{11}$

In addition to technical improvements in the Amrein-Pearson results, we feel that our approach is transparent physically and simple mathematically; indeed, it is so simple that in the end the basic problem appears almost trivial! In part, this is because our method is quite geometric. ${ }^{12}$

To describe the idea consider first potential scattering with $H_{0}=-\frac{1}{2} \nabla^{2}, H=H_{0}+V$, where our basic equation is the following:

$$
\|(S-1) g\|^{2}=\int_{0}^{\infty} \sigma_{\text {tot }}(k)|\hat{g}(k)|^{2} d k \text {. }
$$

In Eq. (1), $\sigma_{\text {to }}(k)$ is the total cross section for initial momentum $(0,0, k), S$ is the $S$ matrix, $g$ is a function of $z$ alone whose Fourier transform $\hat{g}$ is nonzero only for positive $k$, and

$$
\|h\|^{2}=\int|h(x, y, z)|^{2} d x d y d z .
$$

Technically, the left-hand side of (1) is not defined since $g$ is not square integrable, but we intend it to mean ${ }^{13} \lim _{R \rightarrow \infty}\left\|(S-1) g h_{R}\right\|^{2}$ with $h_{R}(x$, $y, z)=\exp \left[-\left(x^{2}+y^{2}\right) / R^{2}\right]$. A moment's reflection will convince one that Eq. (1) is suitable as a definition of $\sigma_{\text {tot }}(k)$. If one prefers the time-independent definition, a simple calculation verifies (1) so long as $V$ is sufficiently short range for the kernel of the on-shell $T$ matrix to be continuous in $k, k^{\prime}$, and energy.

We next write ${ }^{14}$

$$
\begin{aligned}
\|(S-1) g\|=\left\|\left(\Omega^{-}\right) *\left(\Omega^{+}-\Omega^{-}\right) g\right\| & \\
\leqslant\left\|\left(\Omega^{+}-\Omega^{-}\right) g\right\| & =\left\|\int_{-\infty}^{\infty}\left[\exp (+i t H) V \exp \left(-i t H_{0}\right) g\right] d t\right\| \\
& \leqslant \int_{-\infty}^{\infty}\left\|V \exp \left(-i t H_{0}\right) g\right\| d t \\
& =\int_{-\infty}^{\infty} d t\left[\int_{-\infty}^{\infty} d z W(z)^{2}|g(z, t)|^{2}\right]^{1 / 2},
\end{aligned}
$$

with $W(z)=\left[\int d x d y|V(x, y, z)|^{2}\right]^{1 / 2}$ and $g(z, t)=\left[\exp \left(-i t H_{0}\right) g\right](z)$. We choose $g$ so that $\hat{g}$ is smooth and is nonzero only within an interval $\left(k_{0}, k_{0}+\delta\right)$ (with $k_{0}>0$ ) of velocities. For $z / t$ in the classically allowed range $\left(k_{0}, k_{0}+\delta\right)$ we use the estimate

$$
|g(z, t)| \leqslant C t^{-1 / 2}
$$

of one-dimensional wave-packet spreading, and in the classically forbidden region we use Hömander's estimate ${ }^{15}$

$$
|g(z, t)| \leqslant D_{n}(1+|z|+|t|)^{-n} \quad\left(z / t \leqslant k_{0} \text { or } z / t \geqslant k_{0}+\delta\right) .
$$

A typical bound that results is

$$
\int_{k_{0}+\delta / 3}^{k_{0}+2 \delta / 3} \sigma_{\text {tot }}(k) d k \leqslant C\left(k_{0}, \delta, \epsilon\right) \int_{-\infty}^{\infty}(1+|z|)^{1+\epsilon} W(z)^{2} d z .
$$

The right-hand side of (5) is finite if ${ }^{16}|V(\vec{r})| \leqslant(1+|\vec{r}|)^{-2-\epsilon}$ for $|\vec{r}|$ large.

Using multichannel scattering theory, the approach to two-cluster scattering is virtually identical except that formula (3) is replaced by

$$
\int_{-\infty}^{\infty}\left\|I \exp \left(-i t H_{0}\right) g \eta\right\| d t=\int_{-\infty}^{\infty}\left\|V_{\text {eff }} \exp \left(-i t H_{0}\right) g\right\|^{\prime} d t
$$


In Eq. (6), I represents the sum of all potentials between the clusters, $\|\cdots\|$ involves integration over all coordinates, $g$ is a function only of the $z$ component of the separation $\vec{R}$ of the centers of mass, and $\eta$ is the product of the wave functions, $\eta_{1}\left(\rho_{1}\right)$ and $\eta_{2}\left(\rho_{2}\right)$, of the bound clusters. On the right-hand side, $\|\cdots\|^{\prime}$ indicates only an integration over $\vec{R}$ and the effective potential is defined by

$$
V_{\text {eff }}(\overrightarrow{\mathrm{R}})=\left[\int\left|I\left(\overrightarrow{\mathrm{R}}, \rho_{1}, \rho_{2}\right)\right|^{2}\left|\eta_{1}\left(\rho_{1}\right)\right|^{2}\left|\eta_{2}\left(\rho_{2}\right)\right|^{2} d \rho_{1} d \rho_{2}\right]^{1 / 2} \text {. }
$$

Our results on scattering of clusters of charged particles now follow, since in either case $V_{\text {eff }}(\vec{R})$ is bounded by $|\overrightarrow{\mathrm{R}}|^{-3}$ at large distances.

We emphasize that $V_{\text {eff }}$ involves unperturbed bound-cluster wave functions and does not include any polarization which is due to virtual excitation of clusters. This has one surprising consequence. We consider one cluster consisting of an atom in an $s$ state and the other cluster a single charged particle of charge $Z$, and we ask about the growth of $\sigma_{\text {tot }}$ for large $Z .^{17}$ Using the fact that $V_{\text {eff }}$ falls exponentially we can ${ }^{1}$ bound this by $C(\ln Z)^{2}$. Naively one might imagine that a polarization potential going like $Z^{2} R^{-4}$ at large distances might be relevant. For such a two-body potential, even if we use it only for large distances $R>Z^{2 / 3}$, we can only prove a bound as $Z^{4 / 3}$ as $Z \rightarrow \infty$ and we believe that such a potential surely leads to a cross section with power growth as $Z \rightarrow \infty$. Since our $(\ln Z)^{2}$ bound is rigorous, the polarization-potential picture is misleading in this case.

To understand this observe that $\|(S-1) g\|$ meas ures how $\exp (-i t H)$ and $\exp \left(-i t H_{0}\right)$ differ on $g$, which can be estimated by (2), (3), and (6). The relevant interaction is the potential as seen by the state under the free time evolution. In the multiparticle case this is the trivial stationary "motion" within the clusters and the free motion of the centers of mass of the clusters relative to each other. The intercluster forces which are responsible for polarization are "turned off." These effects may show up in bounds on $\sigma_{\text {tot }}$ only if one considers better estimates of (2) than (3).

Alternatively one can estimate

$$
\begin{aligned}
\|(S-1) g\| & =\left\|\left[\left(\Omega^{-}\right)^{*}-\left(\Omega^{+}\right) *\right] \Omega^{+} g\right\| \\
& =\left\|\int d t \exp \left(i t H_{0}\right) V \exp (-i t H) \Omega^{+} g\right\| \\
& \leqslant \int d t\left\|V e^{-i t H} \Omega^{+} g\right\| .
\end{aligned}
$$

If $t=0$ is set long before the scattering takes place, then $\Omega^{+} g \approx g$. Here the potential as seen by the interacting state matters. In the multiparticle case for certain times the polarization potential may be a good effective potential. As the state approaches the scatterer, however, for strong coupling rearrangements will occur, and it looks hopeless to control (8) and everi more to estimate the subtle cancellations which are lost in going from (7) to (8). That cancellations are very important can be seen from the fact that

$$
S-1=\left(\Omega^{-}\right) *(-i) \int_{-\infty}^{\infty} d t \exp (i H t) V \exp \left(-i H_{0} t\right)
$$

is a bounded operator represented as an infinite integral of generally unbounded operators.

Physically, the bound (3) can be interpreted as considering all particles which interact, even only virtually, as being scattered. ${ }^{18}$ Our geometric bounds below come from counting up all particles which enter a sphere about the region of interaction.

In $\nu$-dimensional potential scattering we prove that for an arbitrary positive potential, nonvanishing only for $|x|<R$, the total cross section

$$
\int_{k_{0}}^{k_{0}+\delta}\left|\sigma_{\mathrm{tot}}(k)\right|^{2} d k
$$

is bounded by

$$
C\left(\nu, k_{0}, \delta\right)\left[R^{\nu-1}+\left(R^{\nu-2}\right)\right],
$$

and for potentials of either sign

$$
D\left(\nu, k_{0}, \delta\right)\left[R^{\nu-1}+R^{\nu-3}+\left(R^{\nu-4}\right)\right] .
$$

The terms in parentheses represent terms which are, at the moment, in our estimate but which we believe are not necessary and which we hope to eliminate. We note that for negative potentials, one cannot do better than $R^{\nu-3}$ for small $R$, since in three dimensions, there are point potentials leading to finite cross sections.

In three dimensions, we have proven ${ }^{1}$ that for potentials $V$ obeying $|V(r)| \leqslant D(1+|r|)^{-\alpha}(\alpha>2)$, the cross section for $-\Delta+g V$ in an interval $\left(v_{0}\right.$, $\left.v_{0}+\delta v\right)$ is dominated for $g$ large ${ }^{19}$ by $C(g / v)^{\gamma}$ with $\gamma=2 / \alpha-1$, and, if $|V(r)| \leqslant D e^{-A r}$, by $[\ln (g / v)]^{2}$.

It is a pleasure to thank $\mathrm{W}$. Thirring for having emphasized that there should be a direct physical approach to the problem and P. Deift for valuable discussions. One of us (V.E.) would like to thank the Institute for Advanced Study for its hospitality and support under the Albert Einstein visiting professorship endowed by the Federal Republic of Germany and for a travel grant provided by Deutsche Forschungsgemeinschaft. Another of us (B.S.) acknowledges partial support by the National Science Foundation under Grant No. MCS 78-01885. 
(a) On leave from the Department of Physics, University of Bielefeld, Bielefeld, Federal Republic of Germany.

${ }^{1}$ V. Enss and B. Simon, "Finite Total Cross Sections in Nonrelativistic Quantum Mechanics" (to be published).

${ }^{2} \mathrm{We}$ intend this to mean that there are only Coulomb potentials but additional short-range potentials $\left[O\left(r^{-2-\epsilon}\right)\right.$, $\epsilon>0]$ are allowed without any significant change in our results.

${ }^{3}$ If the cluster has inversion symmetry, this will be the case unless there is a degeneracy of states of different parity.

${ }^{4} \mathrm{By}$ this we mean that if our bound is $\mathrm{Cg}^{\gamma}, \gamma$ is correct asymptotically, but not $C$.

${ }^{5}$ Our constants will be especially bad if the energy is low or the interval of averaging small.

${ }^{6}$ There is, at present, no definitive time-independent approach for multiparticle Coulomb systems.

${ }^{7}$ Except in the region where the Born series converges.

${ }^{8}$ W. O. Amrein and D. B. Pearson, J. Phys. A $\underline{12}$, 1469-1492 (1979).

${ }^{9}$ In addition, A. Martin (CERN Report No. TH 2662 (to be published) $\rfloor$ has obtained a bound going for $g$ large as $g^{4}$ for central potentials. For general $r^{-2-\epsilon}$ potentials we and Amrein and Pearson, Ref. 8 , get a $g^{2}$ bound for large $g$. Also J. M. Combes and A. Tip (private communication from Combes) have informed us that they have a proof of (1) and (2) by different means.

${ }^{10}$ W. O. Amrein, D. B. Pearson, and K. B. Sinha, Nuovo Cimento 52A, 115-131 (1979).

${ }^{11}$ While we have not checked it in detail, we expect that the methods of Refs. 8 and 10 could be extended to handle atom-atom scattering also.

${ }^{12}$ Geometric ideas have been used for some time in rigorous scattering and spectral analysis ; see especially, R. Haag, Phys. Rev. 112, 669-673 (1958); G. M. Zhislin, Mosk. Mat. Obs. 9, 81-128 (1960);
P. D. Lax and R. S. Phillips, Scattering Theory (Academic, New York, 1967); A. G. Sigalov and I. M. Sigal, Teor. Mat. Fiz. 5, 73-93 (1970) [ Theor. Math. Phys. $5,990-1005(1970)]\}$, but they tended to be overshadowed by the power of time-independent methods. Recently, geometric methods have been shown to be extremely powerful in their own right, see, e.g., V. Enss, Commun. Math. Phys. 52, 233 (1977), and 61, 285 (1978); B. Simon, Commun. Math. Phys. 55, 259 (1977), and 58, 205 (1978).

${ }^{13}$ In all steps below the $h_{R}$ can be carried along and $R$-independent bounds easily obtained. Moreover, the estimates show that $\lim _{R \rightarrow \infty}\left\|(S-1) g h_{R}\right\|$ exists.

${ }^{14}$ In potential scattering, the first inequality is actually an equality as a result of asymptotic completeness. In multiparticle systems, equality should hold, but since we do not know rigorously that asymptotic completeness holds we use the inequality which is always true. Equation (2) is just an interaction-picture formula for $S-1$.

${ }^{15}$ L. Hörmander, Math. Zeit 146, 69-91 (1976); see also M. Reed and B. Simon, in Methods of Modern Mathematical Physics (Academic, New York, 1979), Vol. III. The original idea goes back to W. Brenig and R. Haag, Fortschr. Phys. 7, 183-242 (1959).

${ }^{16}$ There is an asymmetry (as there should be) between requirements on falloff in the $z$ and $x-y$ directions. Actually, all that is needed is

$$
|V(x, y, z)| \leqslant C(1+|x|)^{-1 / 2-\epsilon}(1+|y|)^{-1 / 2-\epsilon}(1+|z|)^{-1-\epsilon} .
$$

${ }^{17}$ We do not take into account the effect of changing the mass of the charge $-Z$ projectile in our discussion.

${ }^{18}$ The possible effects of interference between what has been scattered out and what remains in is accommodated by using $\left(\int\|\| d t\right)^{2}$ rather than $\int\|\|^{2} d t$.

${ }^{19}$ Using methods from F. Calogero, Variable Phase Approach to Potential Scattering (Academic, New York, 1967), we have proven that for central potentials obeying $(1+\gamma)^{-\alpha} \leqslant V(r) \leqslant A(1+\gamma)^{-\alpha}$ there is a lower bound behaving as $g^{\gamma}$.

\title{
Turbulent Modification of the $m=1$ Resistive Tearing Instability
}

\author{
A. K. Sundaram and A. Sen \\ Physical Research Laboratory, Ahmedabad 380009, India
}

(Received 16 May 1979)

In the presence of a random spectrum of lower hybrid waves, the $m=1$ resistive tearing instability becomes an oscillating instability with a significantly enhanced growth rate. For typical tokamak parameters, the growth time can become comparable to plasma disruption times for rather moderate levels of fluctuations.

Tearing modes are the subject of intense theoretical investigations these days because of their importance in tokamak plasmas. ${ }^{1}$ They comprise an important class of ideal magnetohydrodynamic
(MHD) modes of the internal kink type for which the perturbations become resonant at the mode rational surface, where $\overrightarrow{\mathrm{k}} \cdot \overrightarrow{\mathrm{B}}_{0}=0$. In this region, it becomes necessary to take into account inertia 